\title{
An Interior Inverse Problem for the Diffusion Operator
}

\author{
A. Dabbaghian, ${ }^{1}$ H. Jafari, ${ }^{2}$ and N. Yosofi ${ }^{3}$ \\ ${ }^{1}$ Islamic Azad University, Neka Branch, P.O. Box 48411-86114, Neka, Iran \\ ${ }^{2}$ Department of Mathematics, University of Mazandaran, P.O. Box 47416-95447, Babolsar, Iran \\ ${ }^{3}$ Department of Mathematics, Islamic Azad University, Sari Branch, Sari, Iran
}

Correspondence should be addressed to H. Jafari; jafari@umz.ac.ir

Received 27 April 2013; Accepted 4 June 2013

Academic Editor: Dumitru Baleanu

Copyright (C) 2013 A. Dabbaghian et al. This is an open access article distributed under the Creative Commons Attribution License, which permits unrestricted use, distribution, and reproduction in any medium, provided the original work is properly cited.

An inverse problem for the diffusion operator on a finite interval with discontinuities conditions inside the interval is studied. We have shown that the potential function of the diffusion operator can be established uniquely by a set of values of eigenfunctions at the midpoint of the interval $(0, \pi)$ and one spectrum.

\section{Introduction}

In an inverse spectral problem, one seeks to determine coefficients in a differential operator from information about the spectrum of the operator, subject to specific side conditions. These kinds of problems arise in a remarkable variety of applications, for example, geophysics, seismology, seismic tomography, optics, and graph theory (see [1-7]).

We consider the boundary value problem $L\left(q_{0}(x), q_{1}(x)\right.$, $h, H)$ of the form

$$
l y(x):=y^{\prime \prime}(x)+\left(\rho^{2}-2 \rho q_{1}(x)-q_{0}(x)\right) y(x)=0
$$

on the interval $0<x<\pi$ with the boundary conditions

$$
\begin{gathered}
U(y):=y^{\prime}(0)-h y(0)=0, \\
V(y):=y^{\prime}(\pi)+H y(\pi)=0
\end{gathered}
$$

and with the jump conditions

$$
\begin{gathered}
y\left(\frac{\pi}{2}+0\right)=a_{1} y\left(\frac{\pi}{2}-0\right), \\
y^{\prime}\left(\frac{\pi}{2}+0\right)=a_{1}^{-1} y^{\prime}\left(\frac{\pi}{2}-0\right)+a_{2} y\left(\frac{\pi}{2}-0\right),
\end{gathered}
$$

where $\rho$ is the spectral parameter, $q_{0}(x)$ and $q_{1}(x)$ are real functions in $L^{2}[0, \pi]$, and the numbers $h, H, a_{1}$, and $a_{2}$ are real and $a_{1}>0$. Without loss of generality, we assume that

$$
\int_{0}^{\pi} q_{1}(x) d x=0 .
$$

Boundary value problems with discontinuities inside the interval are extensively studied $[8,9]$. These kinds of problems are often appear in mathematics, mechanics, physics, and other branches of natural sciences. For example, discontinuous inverse problems appear in electronics for constructing parameters of heterogeneous electronic lines with desirable technical characteristics [10-12]. Also, boundary value problems with discontinuities in an interior point appear in geophysical models for oscillations of the Earth (see $[13,14]$ ). Discontinuous inverse problems (in various formulations) have been considered in [15-17] and other works.

The inverse problem for interior spectral data of the differential operator consists in reconstruction of this operator from the known eigenvalues and some information on eigenfunctions at some internal point.

In the later years, interior inverse problems were studied by several authors [18-20]. In particular, research in [20] discussed the inverse problem for Sturm-Liouville operators with discontinuous boundary conditions and proved that the spectral data of parts of two spectra and some information on eigenfunctions at some interior point of the interval $(0, \pi)$ are sufficient to determine the potential. 
The aim of this paper is to study the inverse problem of reconstructing the diffusion operator with discontinuous conditions on the basis of spectral data of a kind: one spectrum and some information on eigenfunctions at the midpoint of the interval $(0, \pi)$.

\section{Auxiliary Assertions}

Before giving the main results of this work, we will mention some results which will be needed later.

Let $\varphi(x, \rho), C(x, \rho)$, and $S(x, \rho)$ be solutions of (1) under the initial conditions $\varphi(0, \rho)=C(0, \rho)=S^{\prime}(0, \rho)=1, \varphi^{\prime}(0$, $\rho)=h, C^{\prime}(0, \rho)=S(0, \rho)=0$ and under the jump conditions (3). For each fixed $x \in[0, \pi]$, the functions $\varphi(x, \rho), C(x, \rho)$, and $S(x, \rho)$ together with their derivatives with respect to $x$ are entire in $\rho$.

Denote

$$
\Delta(\rho):=-V(\varphi) .
$$

The function $\Delta(\rho)$ is called the characteristic function of $L$. The function $\Delta(\rho)$ is entire in $\rho$ of order $1 / 2$, and its zeros coincide with the eigenvalues of $L$.

Denote

$$
Q(x):=\int_{0}^{x} q_{1}(t) d t .
$$

The functions

$$
\cos (\rho x-Q(x)), \quad \frac{\sin (\rho x-Q(x))}{\rho}
$$

form a fundamental system of solutions for the differential equation

$$
y^{\prime \prime}+\frac{q_{1}^{\prime}(x)}{\rho-q_{1}(x)} y^{\prime}+\left(\rho-q_{1}(x)\right)^{2} y=0 .
$$

We rewrite (1) in the form

$$
\begin{aligned}
y^{\prime \prime}+ & \frac{q_{1}^{\prime}(x)}{\rho-q_{1}(x)} y^{\prime}+\left(\rho-q_{1}(x)\right)^{2} y \\
& =\left(q_{0}(x)+q_{1}^{2}(x)\right) y+\frac{q_{1}^{\prime}(x)}{\rho-q_{1}(x)} y^{\prime} .
\end{aligned}
$$

The function $y=C(x, \rho)$ is a solution of the Cauchy problem for (9) with the initial conditions $y(0)=1, y^{\prime}(0)=0$.

By the method of variation of parameters, we deduce that

$$
\begin{aligned}
& C(x, \rho) \\
& =\cos (\rho x-Q(x)) \\
& +\int_{0}^{x} \frac{\sin (\rho(x-t)-Q(x)+Q(t))}{\rho-q_{1}(t)} \\
& \quad \times\left(\left(q_{0}(t)+q_{1}^{2}(t)\right) C(t, \rho)\right. \\
& \left.+\frac{q_{1}^{\prime}(t)}{\rho-q_{1}^{\prime}(t)} C^{\prime}(t, \rho)\right) d t .
\end{aligned}
$$

$$
\begin{aligned}
& \text { For }|\rho| \rightarrow \infty \\
& C(x, \rho)=\cos (\rho x-Q(x))+O\left(\frac{1}{\rho} \exp (|\operatorname{Im} \rho| x)\right) .
\end{aligned}
$$

Substituting this asymptotic into (10), we calculate

$$
\begin{aligned}
& C(x, \rho) \\
& =\cos (\rho x-Q(x)) \\
& \quad+\frac{\sin (\rho x-Q(x))}{2 \rho} \int_{0}^{x}\left(q_{0}(t)+q_{1}^{2}(t)\right) d t \\
& \quad+\frac{1}{2 \rho} \int_{0}^{x}\left(q_{0}(t)+q_{1}^{2}(t)\right) \\
& \quad \times \sin (\rho(x-2 t)-Q(x)+2 Q(t)) d t \\
& \quad+o\left(\frac{1}{\rho} \exp (|\operatorname{Im} \rho| x)\right) .
\end{aligned}
$$

Differentiating (12) with respect to $x$, we get

$$
\begin{aligned}
& C^{\prime}(x, \rho) \\
& =-\rho \sin (\rho x-Q(x)) \\
& \quad+\frac{\cos (\rho x-Q(x))}{2} \int_{0}^{x}\left(q_{0}(t)+q_{1}^{2}(t)\right) d t \\
& \quad+\frac{1}{2} \int_{0}^{x}\left(q_{0}(t)+q_{1}^{2}(t)\right) \\
& \quad \times \cos (\rho(x-2 t)-Q(x)+2 Q(t)) d t \\
& \quad+o(\exp (|\operatorname{Im} \rho| x)) .
\end{aligned}
$$

Analogously, one can obtain for the function $S(x, \rho)$

$$
\begin{aligned}
S(x, \rho)= & \frac{\sin (\rho x-Q(x))}{\rho}-\frac{\cos (\rho x-Q(x))}{2 \rho^{2}} \\
& \times \int_{0}^{x}\left(q_{0}(t)+q_{1}^{2}(t)\right) d t \\
& +\frac{1}{2 \rho^{2}} \int_{0}^{x}\left(q_{0}(t)+q_{1}^{2}(t)\right) \\
& \quad \times \cos (\rho(x-2 t)-Q(x)+2 Q(t)) d t \\
& +o\left(\frac{1}{\rho^{2}} \exp (|\operatorname{Im} \rho| x)\right),
\end{aligned}
$$




$$
\begin{aligned}
S^{\prime}(x, \rho)= & \cos (\rho x-Q(x)) \\
& -\frac{\sin (\rho x-Q(x))}{2 \rho} \int_{0}^{x}\left(q_{0}(t)+q_{1}^{2}(t)\right) d t \\
& -\frac{1}{2 \rho} \int_{0}^{x}\left(q_{0}(t)+q_{1}^{2}(t)\right) \\
& \times \sin (\rho(x-2 t)-Q(x)+2 Q(t)) d t \\
& +o\left(\frac{1}{\rho} \exp (|\operatorname{Im} \rho| x)\right) .
\end{aligned}
$$

Since $\varphi(x, \rho)=C(x, \rho)+h S(x, \rho)$, by similar arguments in [8], one can calculate, for $|\rho| \rightarrow \infty$,

$$
\begin{aligned}
& \varphi(x, \rho)=\cos (\rho x-Q(x)) \\
& +\left(h+\frac{1}{2} \int_{0}^{x}\left(q_{0}(t)+q_{1}^{2}(t)\right) d t\right) \\
& \times \frac{\sin (\rho x-Q(x))}{\rho} \\
& +o\left(\frac{1}{\rho} \exp (|\operatorname{Im} \rho| x)\right), \quad x<\frac{\pi}{2}, \\
& \varphi(x, \rho)=b_{1} \cos (\rho x-Q(x)) \\
& +b_{2} \cos \left(\rho(\pi-x)-2 Q\left(\frac{\pi}{2}\right)+Q(x)\right) \\
& +M(x) \frac{\sin (\rho x-Q(x))}{2 \rho} \\
& +N(x) \frac{\sin (\rho(\pi-x)-2 Q(\pi / 2)+Q(x))}{2 \rho} \\
& +o\left(\frac{1}{\rho} \exp (|\operatorname{Im} \rho| x)\right), \quad x>\frac{\pi}{2} \\
& \varphi^{\prime}(x, \rho)=-\rho \sin (\rho x-Q(x)) \\
& +\left(h+\frac{1}{2} \int_{0}^{x}\left(q_{0}(t)+q_{1}^{2}(t)\right) d t\right) \\
& \times \cos (\rho x-Q(x)) \\
& +o(\exp (|\operatorname{Im} \rho| x)), \quad x<\frac{\pi}{2}, \\
& \varphi^{\prime}(x, \rho)=\rho\left(-b_{1} \sin (\rho x-Q(x))\right. \\
& \left.+b_{2} \sin \left(\rho(\pi-x)-2 Q\left(\frac{\pi}{2}\right)+Q(x)\right)\right) \\
& +M(x) \frac{\cos (\rho x-Q(x))}{2} \\
& -N(x) \frac{\cos (\rho(\pi-x)-2 Q(\pi / 2)+Q(x))}{2} \\
& +o(\exp (|\operatorname{Im} \rho| x)), \quad x>\frac{\pi}{2},
\end{aligned}
$$

where

$$
\begin{array}{r}
M(x)=b_{1}\left(2 h+\int_{0}^{x}\left(q_{0}(t)+q_{1}^{2}(t)\right) d t\right)+a_{2}, \\
N(x)=b_{2}\left(2 h-\int_{0}^{x}\left(q_{0}(t)+q_{1}^{2}(t)\right) d t\right. \\
\left.+2 \int_{0}^{\pi / 2}\left(q_{0}(t)+q_{1}^{2}(t)\right) d t\right)-a_{2} .
\end{array}
$$

It follows from (6), (17), and (19) that

$$
\begin{aligned}
& \Delta(\rho) \\
& =b_{1}\left[\rho\left(\sin \rho \pi+\frac{b_{2}}{b_{1}} \sin 2 Q\left(\frac{\pi}{2}\right)\right)-\omega \frac{\cos \rho \pi}{2}+\frac{\omega_{1}}{2}\right] \\
& \quad+o(\exp (|\operatorname{Im} \rho| x)),
\end{aligned}
$$

where

$$
\begin{gathered}
\omega=2 H+2 h+\int_{0}^{\pi}\left(q_{0}(t)+q_{1}^{2}(t)\right) d t+\frac{a_{2}}{b_{1}}, \\
\omega=-\left[\frac { b _ { 2 } } { b _ { 1 } } \left(2 H-2 h+\int_{0}^{\pi}\left(q_{0}(t)+q_{1}^{2}(t)\right) d t\right.\right. \\
\left.\left.\quad-2 \int_{0}^{\pi / 2}\left(q_{0}(t)+q_{1}^{2}(t)\right) d t\right)+\frac{a_{2}}{b_{1}}\right] \cos 2 Q\left(\frac{\pi}{2}\right) .
\end{gathered}
$$

Using (21) by the well-known method (see, e.g., [3]), one has that, for $n \rightarrow \infty$,

$$
\rho_{n}=n+\omega_{0}+\frac{1}{2 n \pi}\left(\omega+(-1)^{n-1} \omega_{1}\right)+o\left(\frac{1}{n}\right)
$$

where

$$
\omega_{0}=(-1)^{n-1} \frac{b_{2}}{b_{1} \pi} \sin 2 Q\left(\frac{\pi}{2}\right) .
$$

\section{Main Result}

In this section, we will give a uniqueness theorem. It says that the potential function $q_{0}(x)$ for a diffusion operator is uniquely determined by one spectrum and some information on eigenfunctions at the midpoint of the interval $(0, \pi)$. The technique we used is similar to those used in $[6,9]$.

Together with $L$, we consider a boundary value problem $\widetilde{L}=L\left(\widetilde{q_{0}}, q_{1}, h, H\right)$ of the same form but with a different coefficient $\widetilde{q_{0}}$. We agree that, if a certain symbol $\alpha$ denotes an object related to $L$, then $\widetilde{\alpha}$ will denote an analogous object related to $\widetilde{L}$.

Consider the problems

$$
\varphi^{\prime \prime}+\left(\rho^{2}-2 \rho q_{1}(x)-q_{0}(x)\right) \varphi=0,
$$


with the initial conditions $\varphi(0)=1, \varphi^{\prime}(0)=h$ and

$$
\tilde{\varphi}^{\prime \prime}+\left(\rho^{2}-2 \rho q_{1}(x)-\tilde{q}_{0}(x)\right) \tilde{\varphi}=0,
$$

with the initial conditions $\widetilde{\varphi}(0)=1, \widetilde{\varphi}^{\prime}(0)=h$.

For $x \leq \pi / 2$, the following representation holds (see [21]):

$$
\begin{aligned}
\varphi(x, \rho)= & \cos (\rho x-\alpha(x))+\int_{0}^{x} A(x, t) \cos \rho t d t \\
& +\int_{0}^{x} B(x, t) \sin \rho t d t, \\
\widetilde{\varphi}(x, \rho)= & \cos (\rho x-\alpha(x))+\int_{0}^{x} \widetilde{A}(x, t) \cos \rho t d t \\
& +\int_{0}^{x} \widetilde{B}(x, t) \sin \rho t d t,
\end{aligned}
$$

where

$\alpha(x)$

$$
=x p(0)+2 \int_{0}^{x}[A(\zeta, \zeta) \sin \alpha(\zeta)-B(\zeta, \zeta) \cos \alpha(\zeta)] d \zeta .
$$

The kernels $A(x, t)$ and $B(x, t)$ are the solution of the problem

$$
\begin{gathered}
\frac{\partial^{2} A(x, t)}{\partial x^{2}}-2 p(x) \frac{\partial B(x, t)}{\partial t}-q(x) A(x, t)=\frac{\partial^{2} A(x, t)}{\partial t^{2}}, \\
\frac{\partial^{2} B(x, t)}{\partial x^{2}}+2 p(x) \frac{\partial A(x, t)}{\partial t}-q(x) B(x, t)=\frac{\partial^{2} B(x, t)}{\partial t^{2}} \\
q(x)=-p^{2}(x) \\
+2 \frac{d}{d x}[A(x, x) \cos \alpha(x)+B(x, x) \sin \alpha(x)] \\
A(0,0)=h, \quad B(x, 0)=0, \\
\left.\frac{\partial A(x, t)}{\partial t}\right|_{t=0}=0, \quad \alpha(x)=\int_{0}^{x} p(t) d t .
\end{gathered}
$$

Hence,

$$
\begin{aligned}
\varphi \widetilde{\varphi}=\frac{1}{2}\{1 & +\cos 2[\rho x-\alpha(x)] \\
& \left.+\int_{0}^{x} H(x, \tau) \cos 2[\rho \tau-\alpha(\tau)]\right\} d \tau,
\end{aligned}
$$

where

$$
\begin{aligned}
H(x, t)=2[A & A(x, x-2 \tau)+\widetilde{A}(x, x-2 \tau) \\
& +B(x, x-2 \tau)+\widetilde{B}(x, x-2 \tau) \\
& +\int_{-x+2 \tau}^{x} A(x, s) \widetilde{A}(x, s-2 \tau) d s \\
& +\int_{-x+2 \tau}^{x} B(x, s) \widetilde{B}(x, s-2 \tau) d s \\
& +\int_{-x}^{x-2 \tau} A(x, s) \widetilde{A}(x, s+2 \tau) d s \\
& \left.+\int_{-x}^{x-2 \tau} B(x, s) \widetilde{B}(x, s+2 \tau) d s\right] .
\end{aligned}
$$

The eigenvalues and the corresponding eigenfunctions of the problem $L$ are denoted by $\rho_{n}$ and $\varphi_{n}(x), n \in N$, respectively.

Theorem 1. If for any $n \in N$,

$$
\rho_{n}=\widetilde{\rho}_{n}, \quad\left[\varphi_{n}, \widetilde{\varphi}_{n}\right]\left(\frac{\pi}{2}-0\right)=0,
$$

then $q_{0}=\widetilde{q}_{0}$ almost everywhere on $[0, \pi]$.

Proof. If we multiply (25) by $\widetilde{\varphi}$ and (26) by $\varphi$, and then subtract, after integrating on $[0,(\pi / 2)-0]$, we obtain

$$
\begin{aligned}
D & (\rho) \\
& :=\int_{0}^{(\pi / 2)-0}[\tilde{q}(x)-q(x)] \varphi(x, \rho) \tilde{\varphi}(x, \rho) d x \\
& =\left.\left[\tilde{\varphi}^{\prime}(x, \rho) \varphi(x, \rho)-\tilde{\varphi}(x, \rho) \varphi^{\prime}(x, \rho)\right]\right|_{0} ^{(\pi / 2)-0} .
\end{aligned}
$$

By using the properties of $\varphi$ and $\widetilde{\varphi}$, we conclude that the function $D(\rho)$ is an entire function. From condition of the theorem, together with the initial-value condition at 0 , it follows that $D\left(\rho_{n}\right)=0, n \in N$.

In addition, by (27), (28), and (34), for $0<x<\pi$, we find

$$
|D(\rho)| \leq M,
$$

where $M>0$ is constant. Now, we define an entire function

$$
\Psi(\rho)=\frac{D(\rho)}{\Delta(\rho)} .
$$

From (21) and (35), it follows that

$$
|\Psi(\rho)|=O\left(\frac{1}{\rho}\right),
$$

for large $|\rho|$. So, for all $\rho$, from the Liouville theorem, we get

$$
\Psi(\rho)=0 \text { or } \quad D(\rho)=0 .
$$


Define $r(x)=\widetilde{q}(x)-q(x)$. Further substituting (31) into (34) and (38), we obtain

$$
\begin{aligned}
\frac{1}{2} \int_{0}^{(\pi / 2)-0} r(x)\{ & {[1+\cos 2(\rho x-\alpha(x))] } \\
+ & \left.\int_{0}^{x} H(x, \tau) \cos 2(\rho \tau-\alpha(\tau)) d \tau\right\} d x=0,
\end{aligned}
$$

which can be rewritten as

$$
\begin{aligned}
& \int_{0}^{(\pi / 2)-0} r(x) d x \\
& +\int_{0}^{(\pi / 2)-0} \cos 2(\rho \tau-\alpha(\tau)) \\
& \times\left[r(\tau)+\int_{\tau}^{(\pi / 2)-0} r(x) H(x, \tau) d x\right] d \tau=0 .
\end{aligned}
$$

Letting $\rho \rightarrow \infty$ for real $\rho$, we conclude from RiemannLebesgue lemma that

$$
\begin{gathered}
\int_{0}^{(\pi / 2)-0} r(x) d x=0, \\
\int_{0}^{(\pi / 2)-0} \cos 2(\rho \tau-\alpha(\tau)) \\
\times\left[r(\tau)+\int_{\tau}^{(\pi / 2)-0} r(x) H(x, \tau) d x\right] d \tau=0 .
\end{gathered}
$$

Then, by using the trigonometric expansion of cos function and the completeness of the functions cos and sin, we obtain

$$
r(\tau)+\int_{\tau}^{(\pi / 2)-0} r(x) H(x, \tau) d x=0, \quad 0<\tau<\frac{\pi}{2} .
$$

Since (43) is a Volterra integral equation, it has only trivial solution. Hence, we have obtained our result $r(x)=0$ on $0<$ $x<\pi / 2$; that is, $q_{0}(x)=\widetilde{q}_{0}(x)$ almost everywhere on $[0, \pi / 2]$.

To prove that $q_{0}(x)=\tilde{q}_{0}(x)$ almost everywhere on $[\pi / 2, \pi]$, we will consider the supplementary problem $\widehat{L}$ :

$$
\begin{gathered}
\varphi^{\prime \prime}(x)+\left(\rho^{2}-2 \rho q_{11}(x)-q_{01}(x)\right) \varphi(x)=0, \\
0<x<\pi, \\
U(\varphi):=\varphi^{\prime}(0)-H \varphi(0)=0, \\
V(\varphi):=\varphi^{\prime}(\pi)+h \varphi(\pi)=0, \\
\varphi\left(\frac{\pi}{2}+0\right)=a_{1}^{-1} \varphi\left(\frac{\pi}{2}-0\right), \\
\varphi^{\prime}\left(\frac{\pi}{2}+0\right)=a_{1} \varphi^{\prime}\left(\frac{\pi}{2}-0\right)+a_{1} a_{2} \varphi\left(\frac{\pi}{2}-0\right),
\end{gathered}
$$

where

$$
q_{11}(x)=q_{1}(\pi-x), \quad q_{01}(x)=q_{0}(\pi-x) .
$$

Note that, if $y(x)$ and $z(x)$ satisfy the matching conditions (3), then a direct calculation yields

$$
[y, z]\left(\frac{\pi}{2}+0\right)=[y, z]\left(\frac{\pi}{2}-0\right) .
$$

The assumption of Theorem 1 and (46) imply that

$$
\left[\varphi_{n}, \widetilde{\varphi}_{n}\right]\left(\frac{\pi}{2}+0\right)=0
$$

A direct calculation implies that $\widehat{\varphi}_{n}(x):=\varphi_{n}(\pi-x)$ is the solution to the supplementary problem $\widehat{L}$ and $\widehat{\varphi}_{n}((\pi / 2)-0):=$ $\varphi_{n}((\pi / 2)+0)$. Thus, for the supplementary problem $\widehat{L}$, the assumption conditions in the theorem are still satisfied.

If we repeat the previous arguments, then this yields $r(\pi-x)=0$ on $0<x<\pi / 2$; that is, $q_{0}(x)=\widetilde{q}_{0}(x)$ almost everywhere on $[\pi / 2, \pi]$. The proof of the theorem is finished.

We suggest to extend this work for fractional differential equations and local fractional differential equations [22-24] when the order of $y(x)$ is noninteger in (1).

\section{References}

[1] K. Aki and P. G. Richards, in Quantitative Seismology: Theory and Methods, vol. 1, chapter 8, pp. 337-381, W. H. Freeman, New York, NY, USA, 1980.

[2] W. Rundell and P. E. Sacks, "Reconstruction of a radially symmetric potential from two spectral sequences," Journal of Mathematical Analysis and Applications, vol. 264, no. 2, pp. 354381, 1991.

[3] H. P. Baltes, Inverse Scattering Problems in Optics, vol. 20 of Topics in Current Physics, Springer, Berlin, Germany, 1980.

[4] L. Hogben, "Spectral graph theory and inverse eigenvalue problem of a graph," Chamchuri Journal of Mathematics, vol. 1, no. 1, pp. 51-72, 2009.

[5] C. R. Johnson, A. Leal-Duarte, and C. M. Saiago, "Inverse eigenvalue problems and lists of multiplicities of eigengvalues for matrices whose graph is a tree: the case of generalized stars and double generalized stars," Linear Algebra and Its Applications, vol. 373, pp. 311-330, 2003.

[6] R. L. Parker and K. A. Whaler, "Numerical methods for establishing solutions to theinverse problem of electromagnetic induction," Journal of Geophysical Research, vol. 86, no. 10, pp. 9574-9584, 1981.

[7] V. Yurko, "Uniqueness of recovering differential operators on hedgehog-type graphs," Advances in Dynamical Systems and Applications, vol. 4, no. 2, pp. 231-241, 2009.

[8] G. Freiling and V. A. Yurko, Inverse Sturm-Liouville Problems and Their Applications, Nova Science, New York, NY, USA, 2001.

[9] O. H. Hald, "Discontinuous inverse eigenvalue problems," Communications on Pure and Applied Mathematics, vol. 37, no. 5, pp. 539-577, 1984.

[10] O. N. Livinenko and V. I. Soshnikov, The Theory of Heterogeneous Lines and Their Applications in Radio Engineering, Radio, Moscow, Russia, 1964 (Russian).

[11] J. R. McLaughlin and P. L. Polyakov, "On the uniqueness of a spherically symmetric speed of sound from transmission eigenvalues," Journal of Differential Equations, vol. 107, no. 2, pp. 351-382, 1994. 
[12] V. P. Meschanov and A. L. Feldstein, Automatic Design of Directional Couplers, Sviaz, Moscow, Russia, 1980.

[13] R. S. Anderssen, "The effect of discontinuities in density and shear velocity onthe asymptotic overtone structure of torsional eigenfrequencies of the Earth," Geophysical Journal of the Royal Astronomical Society, vol. 50, pp. 303-309, 1997.

[14] F. R. Lapwood and T. Usami, Free Oscillations of the Earth, Cambridge University Press, Cambridge, UK, 1981.

[15] G. Freiling and V. A. Yurko, "Inverse spectral problems for singular non-selfadjoint differential operators with discontinuities in an interior point," Inverse Problems, vol. 18, no. 3, pp. 757-773, 2002.

[16] R. J. Krueger, "Inverse problems for nonabsorbing media with discontinuous material properties," Journal of Mathematical Physics, vol. 23, no. 3, pp. 396-404, 1982.

[17] V. A. Yurko, "On boundary value problems with discontinuity conditions inside an interval," Differentsial'nye Uravneniya, vol. 36, no. 8, pp. 1139-1140, 2000 (Russian), English Translation in Differential Equations, vol. 8, no. 8, pp. 1266-1269, 2000.

[18] K. Mochizuki and I. Trooshin, "Inverse problem for interior spectral data of the Sturm-Liouville operator," Journal of Inverse and Ill-Posed Problems, vol. 9, no. 4, pp. 425-433, 2001.

[19] K. Mochizuki and I. Trooshin, "Inverse problem for interior spectral data of the Dirac operator on a finite interval," Publicationsof the Research Institute for Mathematical Sciences, Kyoto University, vol. 38, no. 2, pp. 387-395, 2002.

[20] C. F. Yang and X. P. Yang, "An interior inverse problem for the Sturm-Liouville operator with discontinuous conditions," Applied Mathematics Letters, vol. 22, no. 9, pp. 1315-1319, 2009.

[21] M. G. Gasymov and G. Š. Guseǐnov, "Determination of a diffusion operator from spectral data," Akademiya Nauk Azerbaĭdzhanskoĭ SSR. Doklady, vol. 37, no. 2, pp. 19-23, 1981.

[22] D. Baleanu, K. Diethelm, E. Scalas, and J. J. Trujillo, Fractional calculus: models and numerical methods, vol. 3 of Series on Complexity, Nonlinearity and Chaos, World Scientific, Boston, Mass, USA, 2012.

[23] X. J. Yang, Advanced Local Fractional Calculus and Its Applications, World Science, New York, NY, USA, 2012.

[24] X. J. Yang, Local Fractional Functional Analysis and Its Applications, Asian Academic, Hong Kong, China, 2011. 


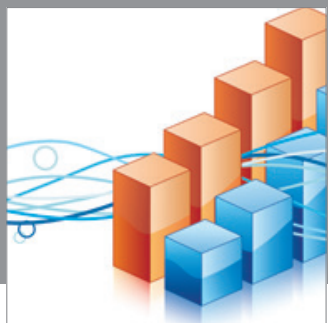

Advances in

Operations Research

mansans

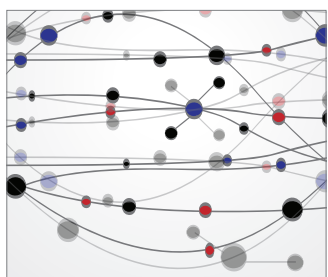

The Scientific World Journal
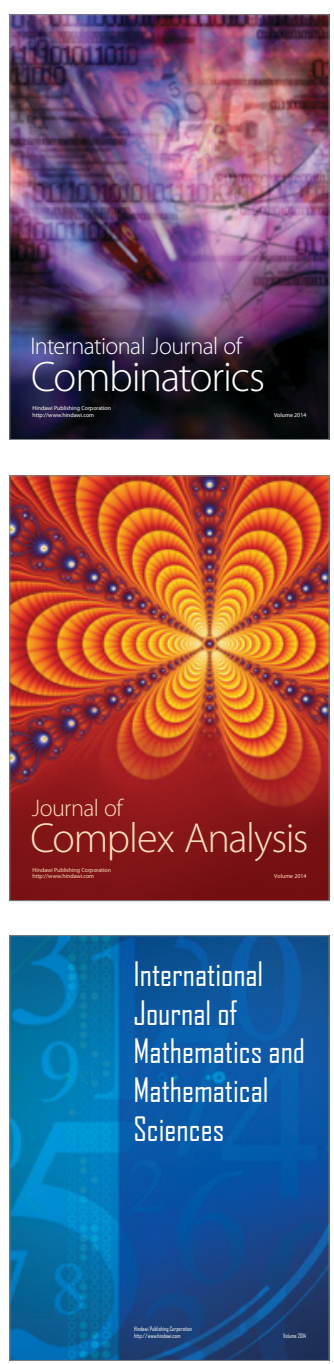
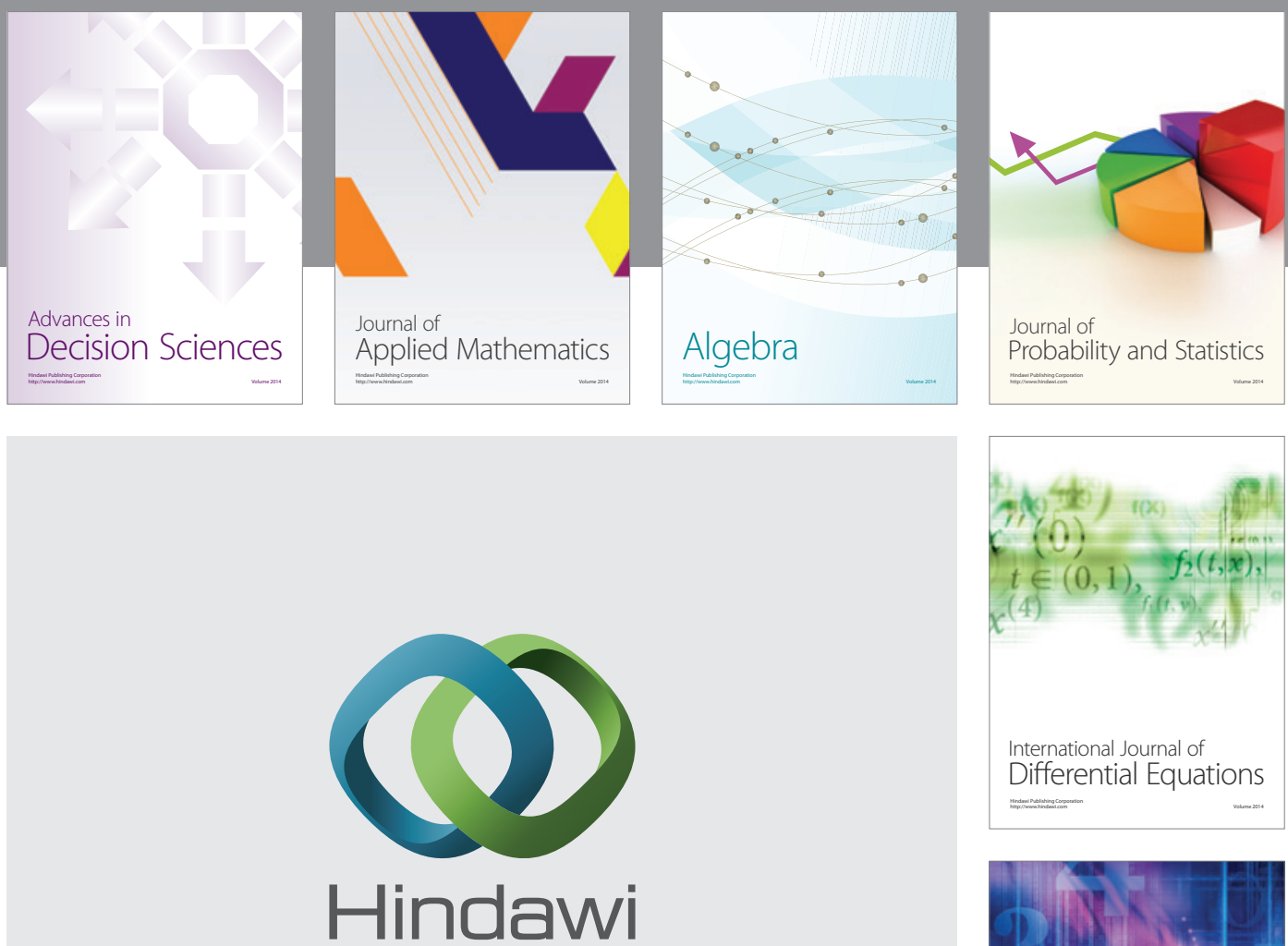

Submit your manuscripts at http://www.hindawi.com
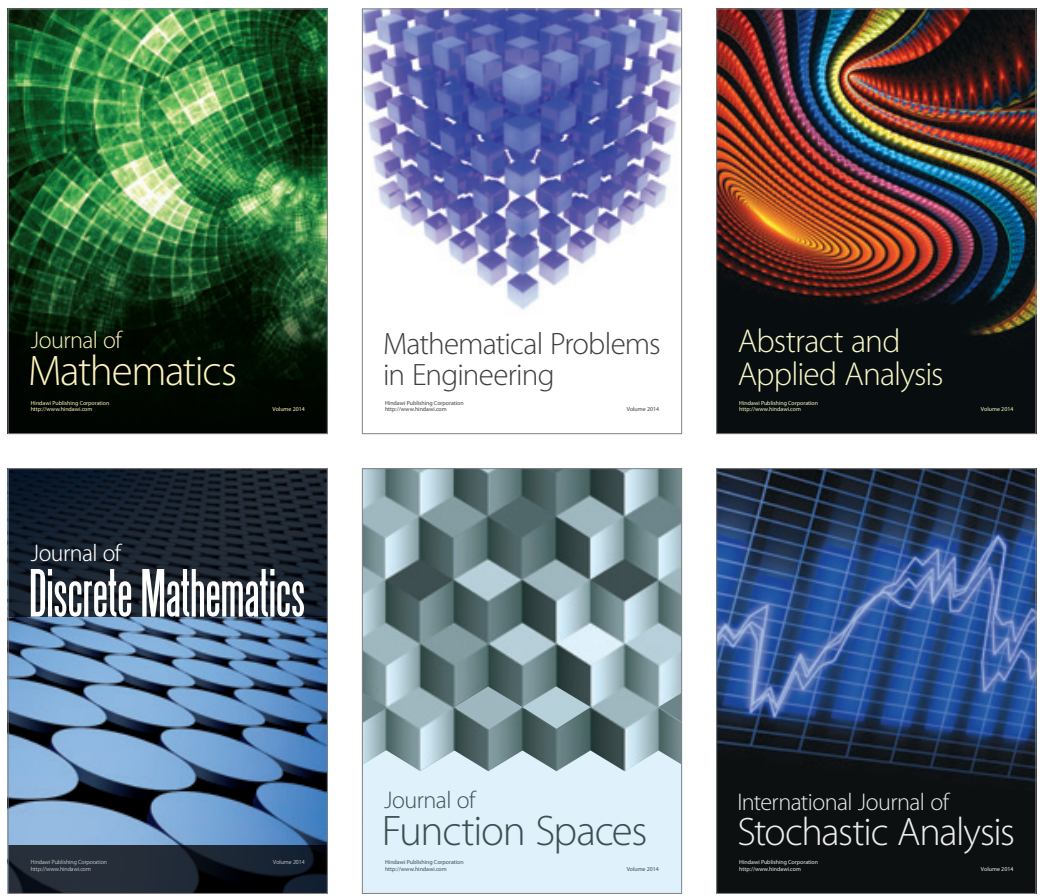

Journal of

Function Spaces

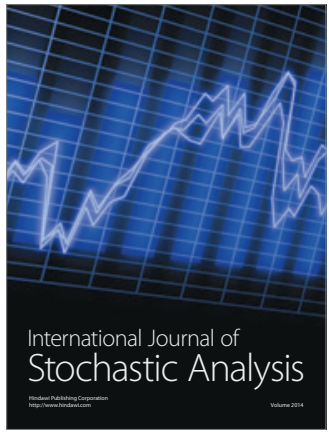

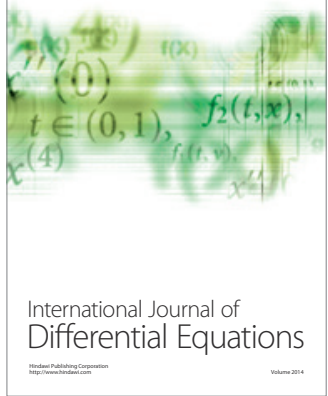
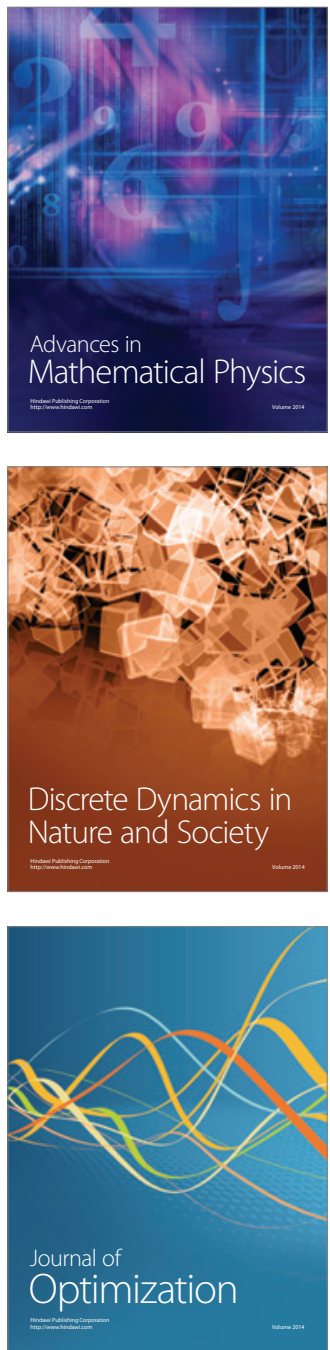